\title{
A RARE SARCOMA DIAGNOSED ON FINE NEEDLE ASPIRATION IN TWO PATIENTS WITH CASTLEMAN DISEASE clinical cases with questions for students
}

\author{
Wendong $\mathrm{Yu}^{1}$, Uma Kundu ${ }^{1}$
}

${ }^{1}$ MD Anderson Cancer Center, Department of Pathology and Laboratory Medicine, Section of Cytopathology, Houston, USA

\section{Case 1:}

A 38 year old female presented with a $3.6 \times 1.8 \mathrm{~cm}$ peri-aortic mass and retroperitoneal lymphadenopathy. She initially presented as anosed on core needle biopsy as hyaline vassuprarenal mass seven years prior which wemotherapy of gemcitabine and the right suprarecular Castleman disease. Afte subsequently underwent surgical The resected specimen showed remission was achieved. Sh at MD Anderson Cancer Center. Thell mature lymphocytes. Many nal mass and right kidney at Momor cells in a background of sme we also areas with prominent large spindle and epithelioin tuclear pseudoinclusions. There weosinophilic extracellular matetumor cells had prominent proliferation of plasma cells, focal dystrophic calcification was present. rial. Additionally, focaldyslin light-chain showed a polytypic pithelioid tumor cells were posiand lambda immunoga lambda light chains. The spindled and cells for kapp for CD21,CD35, clusterin and vimentin by immune 1-3).

aspirate smears and cell block are shown below (image $1-3)$.


Image 1. Pap stained smear, 20x (A), 40x (B) and 60x (C) magnification.

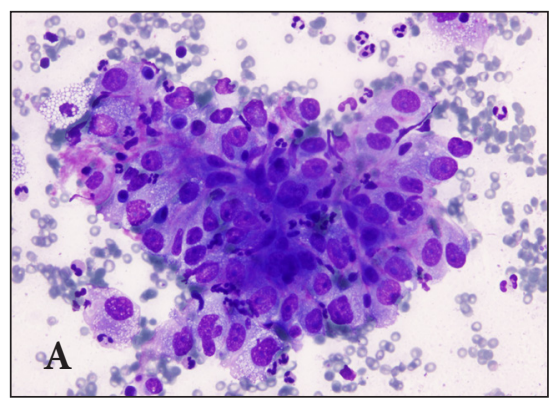

Image 2. Diff-Quik Stain, 20x (A), 40x (B) magnification.

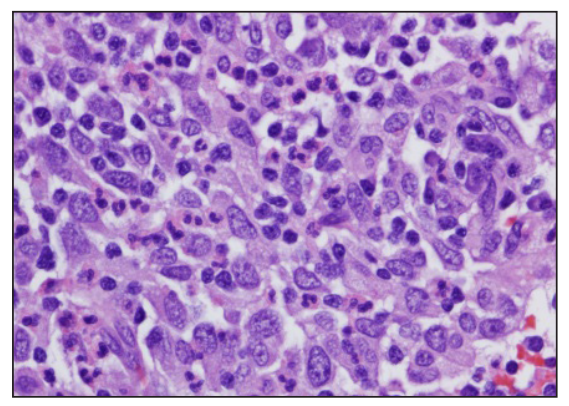

Image 3. Cell block (40x). 


\section{Case 2:}

53 year old male presented with a $6.1 \mathrm{~cm}$, lobulated, well-circumscribed extra-hepatic mass at the level of the porta-hepatis encasing the main portal vein. Hascular Castleman disease. as a peri-gastric soft tissue mass arising a a bactrectomy. The slides from the resected neoplasm were At that time, he underwent a partial Center and showed both spindle cells and resembled hyaline reviewed at MD Andersontologically low-grade and closely related fo CD21, CD35, CXCL13, D2The spindle cells were cysease. The spindled areas were positr. A subset of epithelioid cells was vascular Castleman dise vimentin by immunohistochembridization for Epstein-Barr virus small 40, fascin, clusterin an CD68 immunostains. In-situ markers for T-cells, B-cells, muscle specific positive for $\mathrm{CD}$ (EBER) and immunohistochemical me A CT guided fine needle aspir the images from the aspirate smemical features were simiat MD Anderson Cancer Center and the cytomorphologic and immunhistochemical features we

shown below (image 4-6). Theri-gastric tumor.
lar to the patient's initial peri-

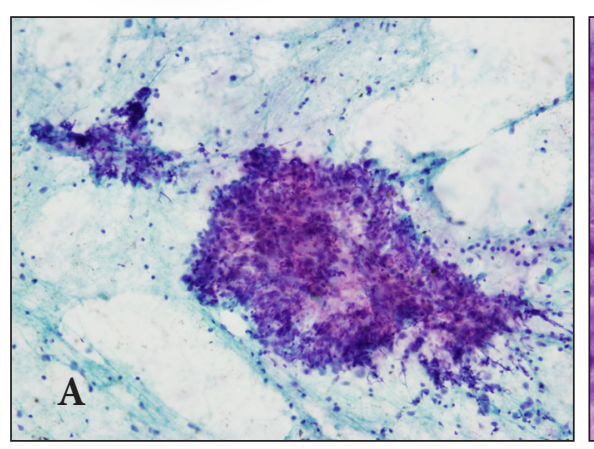

Image 4. Pap stained smear, 20x (A), 40x (B) magnification.



Image 5. Cell block (40x).
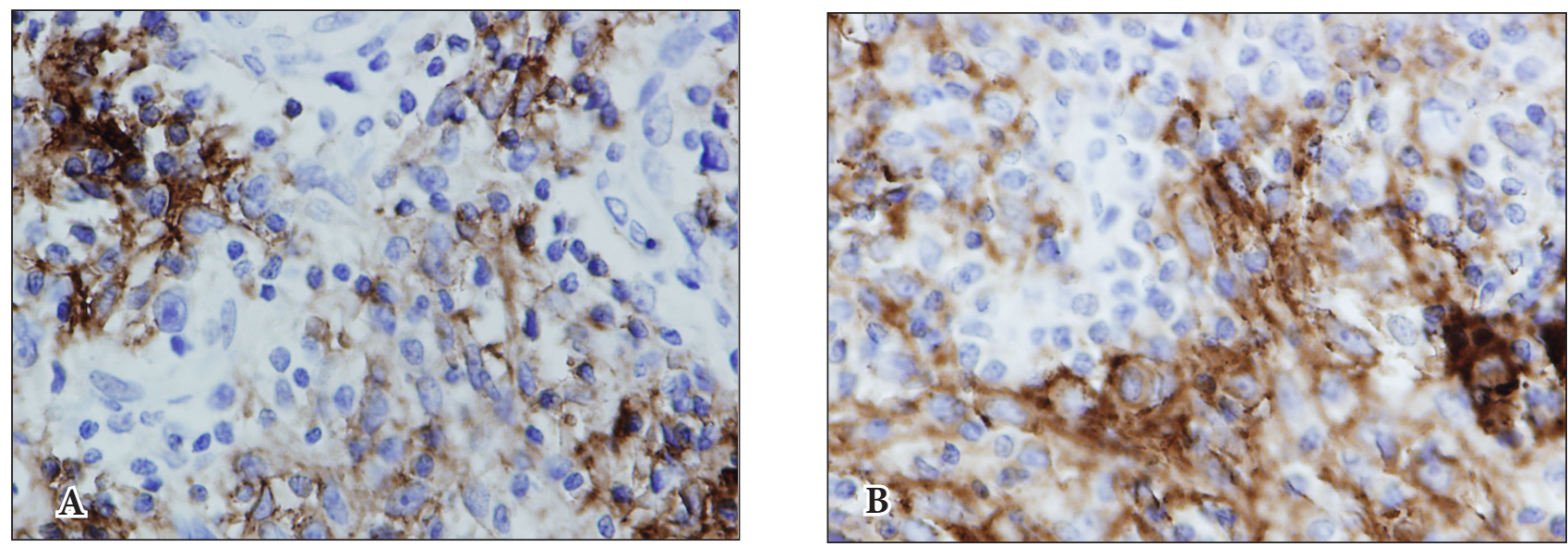

Image 6. Immunostain CD21 (A) and CD35 (B). 
Which is the most likely diagnosis?

$\begin{array}{ll}\text { A. } & \text { Follicular dendritic cell sarcoma } \\ \text { B. Gastrointestinal stromal tumor (GIST) } \\ \text { C. Leimyosarcoma } \\ \text { D. Malignant fibrous histiocytoma (MFH) } \\ \text { E. Melanoma }\end{array}$

The answer is A. Follicular dendritic cell sarcoma

Follicular dendritic cell sarcoma (FDCS) was first described by Monda and coworkers in 1986 [1]. It is a rare neoplasm characterized by a dual cell population of malignant large epithelioid and spindled cells in a background of numerous small lymphocytes. The lymphocytes are of T-cell origin [2]. Interestingly, a recent study shows that $\mathrm{Td} \mathrm{T}+\mathrm{T}$-lymphoblastic populations may be increased in Castleman disease associated with FDCS [3].

FDCS can present in nodal (cervical or mediastinal) or extranodal (skin, soft tissue, tonsil, GI tract liver and spleen) sites. It is a tumor of young adults usually presenting less than 50 years of age with no gender predilection. There is an association with hyaline-vascular Castleman follicular hyperplasia [4] and Epstein-Barr virus in cases associated with inflammatory pseudo-tumor $[5,6]$. Both recurrence and metastasis can occur. Majority of tumor presented as an early disease (85.4\%), while $6.4 \%$ presented as locally advanced disease, and $8.2 \%$ presented with distant metastasis [7]. FDCS is considered a low to intermediate grade tumor with an overall mortality of $17 \%$. When presented as local disease only, median survival is 168 months (range 2-360 months)[7]. The presence of certain histological features such as foci of necrosis and high mitotic activity along with an intra-abdominal location has been correlated with a more aggressive clinical course [8,9]. Additionally, absence of lymphoplasmacytic response and large tumor size also appears to be associated with poor prognosis [7].

Histologically, the tumor is composed of oval to spindle cells forming fascicles, storiform patterns and whorls. The neoplastic cells have plump, slightly eosinophillic cytoplasm with indistinct cell borders and the nuclei are elongated with vesicular or granular finely dispersed chromatin. Occasional multinucleated giant cells and pseudo-intranuclear inclusions may be seen [4]. The presence of small lymphocytes throughout the tumor is characteristic [10]. The mitotic rate is usually between 0 and 10 per 10 high power fields. Uninvolved residual lymphoid tissue is often present in cases with nodal involvement. This may take the form of residual germinal centers or clusters of small lymphocytes in a perivascular location. These tumors may also contain foci of necrosis $[4,11]$.

On cytological examination, both the Diff-Quik and Papanicolaou-stained smears in this case showed that the tumor cells were syncytial and discohesive clusters of epithelioid to spindled cells in a mixed inflammatory background similar to previously described cases of FDCS [2, 10-13]. The tumor cells had eosinophillic granular cytoplasm and indistinct cell borders. The nuclei were usually single with small distinct nucleoli. Although marked pleomorphism, atypical mitotic figures and multi-nucleated giant cells have been reported, we did not observe these features in our cases [11,13]. Intranuclear inclusions and nuclear grooves were present in our cases similar to a previous report [2].

The neoplastic cells in FDCS demonstrate the phenotype of non-neoplastic follicular dendritic cells. They are positive for one or more of the follicular dendritic cell markers, including CD21, CD35 and CD23 [4]. They are also positive for clusterin, desmoplakin, vimentin, fascin, EMA and variably positive for S100 and CD68 $[4,14]$. Ki-67 labeling ranges from 1 to $25 \%$ [4]. BRAF (V600E) mutations can be detected in $18.5 \%$ of FDCS. The significance of this mutation in the pathogenesis of FDCS requires further investigation [15]. Complex cytogenetic abnormalities including losses of multiple chromosomes have been reported in FDCS [16].

The diagnosis can be challenging when cytology is the sole diagnostic modality. The clinical presentation can be variable and the cytomorphological features can overlap with other tumors including sarcomatoid carcinoma, gastrointestinal stomal tumor (GIST), leiomyosarcoma, malignant fibrous histiocytoma (MFH) and melanoma. Distinction from the other tumors is essential because of the low to intermediate grade nature of FDCS. Sarcomatoid carcinoma, also called spindle cell carcinoma, is characterized by anaplastic spindle cells with prominent necrosis and mitotic activity, features usually not seen in FDCS. The carcinoma cells are positive for cytokeratin. GIST is characterized by a prolif- 
eration of bland spindle cells with whorls and short intersecting fascicles. The tumor cells in GIST stain with CD117 which is not observed in FDCS. Leiomyosarcoma is characterized by fascicles of spindle cells intersecting at right angles. The cells have cigar-shaped blunt-ended nuclei with variable atypia and will be positive for muscle markers such as desmin and SMA. The cells of MFH are more pleomorphic with bizarre giant cells and increased mitotic activity in a mixed inflammatory background. While melanoma can have a variety of histological features, the cells are usually pleomorphic with prominent nucleoli. Melanomas will be positive for HMB-45 and S100 without staining for CD21 or CD35. In addition, the presence of melanin pigment would aid in the diagnosis of melanoma.

\section{Check your knowledge}

1. Which of the following features can be seen in cytology smears of FDCS?
A. Syncytial clusters and discohesive cells
B. Spindled and epithelioid morphology
C. Intranuclear inclusions and nuclear grooves
D. Lymphocytes and plasma cells
E. All of the above

The answer is $\mathbf{E}$.

2. Which immunohistochemical markers support a diagnosis of FDCS?
A. CD21 or CD35
B. CD117
C. Vimentin
D. Cytokeratin

The answer is $\mathbf{A}$.

3. What clinical features are characteristic of FDCS?
A. The tumor is more common in women than men.
B. The tumor only presents in elderly patients.
C. Recurrence and metastasis never occur.
D. Presentation in both nodal and extranodal sites has been reported.

The answer is $\mathbf{D}$.

4. Which of the following has been associated with FDCS?
A. Castleman disease
B. Rheumatoid arthritis
C. Sjögren's syndrome
D. Mesothelial hyperplasia
E. Rhabdomyosarcoma

The answer is $\mathbf{A}$.

5. Which of the following features has been associated with a more aggressive clinical course for FDCS?
A. Patient age greater than 50 years
B. Nodal presentation
C. Intranuclear inclusions
D. Absence of mitotic activity
E. Intraabdominal location

The answer is $\mathbf{E}$.

6. Which of the following has been associated with FDCS?
A. Polyoma Virus
B. Ebstein Barr Virus
C. Herpes Virus
D. Enterovirus
E. Human Papilloma Virus

The answser is $\mathbf{B}$.

7. The overall mortality rate for FDCS has been reported as:
A. $90 \%$
B. $60 \%$
C. $40 \%$
D. $17 \%$
E. $5 \%$ 
8. Which of the following statements about FDCS is true?
A. FDCS does not demonstrate the presence of lymphocytes on cytology smears.
B. The differential diagnosis is limited to melano- ma only.
C. No cases of FDCS containing multi-nucleated giant cells have been reported.
D. The neoplastic cells of FDCS can be variably positive for $\mathrm{S} 100$.
E. Ki-67 labeling is usually above $90 \%$.

The answer is $\mathbf{D}$.

\section{References}

1. Monda L, Warnke R, Rosai J: A primary lymph node malignancy with features suggestive of dendritic reticulum cell differentiation. A report of 4 cases. The American journal of pathology 1986, 122:562-72.

2. Gaffney RL, Feddersen RM, Bocklage TJ, et al.: Fine needle aspiration cytology of follicular dendritic cell sarcoma. Report of a case with cytologic detection in an extranodal site. Acta cytologica 2000, 44:809-14.

3. Ohgami RS, Zhao S, Ohgami JK, et al.: TdT+ T-lymphoblastic populations are increased in Castleman disease, in Castleman disease in association with follicular dendritic cell tumors, and in angioimmunoblastic T-cell lymphoma. The American journal of surgical pathology 2012, 36:1619-28.

4. C. F, K. U, M. M: World Health Organisation Classification of Tumors. Pathology and Genetics of Tumours of Soft Tissue and Bone. Lyon, France: IARC Press, 2002.

5. Cossu A, Lissia A, Dedola MF, et al.: Classic follicular dendritic reticulum cell tumor of the lymph node developing in a patient with a previous inflammatory pseudotumor-like proliferation. Human pathology 2005, 36:20711.

6. Granados R, Aramburu JA, Rodriguez JM, et al.: Cytopathology of a primary follicular dendritic cell sarcoma of the liver of the inflammatory pseudotumor-like type. Diagnostic cytopathology 2008, 36:42-6.

7. Saygin C, Uzunaslan D, Ozguroglu M, et al.: Dendritic cell sarcoma: a pooled analysis including 462 cases with presentation of our case series. Critical reviews in oncology/ hematology 2013, 88:253-71.

8. Chan JK, Fletcher CD, Nayler SJ, et al.: Follicular dendritic cell sarcoma. Clinicopathologic analysis of 17 cases suggesting a malignant potential higher than currently recognized. Cancer 1997, 79:294-313.

9. Perez-Ordonez B, Erlandson RA, Rosai J: Follicular dendritic cell tumor: report of 13 additional cases of a distinctive entity. The American journal of surgical pathology 1996, 20:944-55.
10. Kure K, Khader SN, Suhrland MJ, et al.: Fine needle aspiration of follicular dendritic cell sarcoma in an HIV-positive man: a case report. Acta cytologica 2010, 54:707-11.

11. Ren R, Sun X, Staerkel G, et al.: Fine-needle aspiration cytology of a liver metastasis of follicular dendritic cell sarcoma. Diagnostic cytopathology 2005, 32:38-43.

12. Ryley NG, Bastert J, Ferguson DJ, et al.: Follicular dendritic cell sarcoma of lymph node--report of fine needle aspiration (FNA) cytological appearances. Cytopathology: official journal of the British Society for Clinical Cytology 1999, 10:335-40.

13. Tokyol C, Yilmaz MD, Ekici O, et al.: Follicular dendritic cell sarcoma: a case report. Acta cytologica 2008, 52:235-9.

14. Grogg KL, Macon WR, Kurtin PJ, et al.: A survey of clusterin and fascin expression in sarcomas and spindle cell neoplasms: strong clusterin immunostaining is highly specific for follicular dendritic cell tumor. Modern pathology : an official journal of the United States and Canadian Academy of Pathology, Inc 2005, 18:260-6.

15. Go H, Jeon YK, Huh J, et al.: Frequent detection of BRAF(V600E) mutations in histiocytic and dendritic cell neoplasms. Histopathology 2014, 65:261-72.

16. Perry AM, Nelson M, Sanger WG, et al.: Cytogenetic abnormalities in follicular dendritic cell sarcoma: report of two cases and literature review. In vivo (Athens, Greece) 2013, 27:211-4. 